\title{
Approximate Ergodic Capacity of a Class of Fading 2-user 2-hop Networks
}

\author{
Sang-Woon Jeon, Chien-Yi Wang, and Michael Gastpar ${ }^{\dagger}$ \\ School of Computer and Communication Sciences \\ EPFL \\ Lausanne, Switzerland \\ \{sangwoon.jeon, chien-yi.wang, michael.gastpar\}@epfl.ch
}

\begin{abstract}
We consider a fading AWGN 2-user 2-hop network in which the channel coefficients are independently and identically distributed (i.i.d.) drawn from a continuous distribution and vary over time. For a broad class of channel distributions, we characterize the ergodic sum capacity within a constant number of bits/sec/Hz, independent of signal-to-noise ratio. The achievability follows from the analysis of an interference neutralization scheme where the relays are partitioned into $K$ pairs, and interference is neutralized separately by each pair of relays. For $K=1$, we previously proved a gap of $4 \mathrm{bits} / \mathrm{sec} / \mathrm{Hz}$ for i.i.d. uniform phase fading and approximately $4.7 \mathrm{bits} / \mathrm{sec} / \mathrm{Hz}$ for i.i.d. Rayleigh fading. In this paper, we give a result for general $K$. In the limit of large $K$, we characterize the ergodic sum capacity within $4((\log \pi)-1) \simeq 2.6 \mathrm{bits} / \mathbf{s e c} / \mathbf{H z}$ for $\mathbf{i . i . d}$. uniform phase fading and $4(4-\log 3 \pi) \simeq 3.1$ bits/sec/Hz for i.i.d. Rayleigh fading.
\end{abstract}

\section{INTRODUCTION}

In recent years, there has been significant progress towards understanding fundamentals of multi-source single-hop networks. Approximate capacity results have been proved for the 2-user interference channel [1], many-to-one or one-tomany interference channel [2], and two-way channel [3], which characterize the capacity within a constant number of $\mathrm{bits} / \mathrm{sec} / \mathrm{Hz}$ independent of signal-to-noise ratio (SNR) and channel parameters. It has also been proved in [4] that interference alignment can achieve the optimal degrees of freedom (DoF) of the time-varying $K$-user interference channel. The recently proposed ergodic interference aliment in [5] makes interference aligned at finite SNR.

For multi-source multi-hop networks, interference can be cancelled by aligning multiple paths through the network, a technique termed as interference neutralization. Proper use of such interference neutralization is the key for an approximate capacity [6] or DoF characterization [7]-[11] of multi-source multi-hop networks. In spite of recent progress in this area, the best known capacity characterization for fully connected 2-user 2-hop networks is within $o(\log (\mathrm{SNR})) \mathrm{bits} / \mathrm{sec} / \mathrm{Hz}$ [8], which can be arbitrarily large as SNR increases.

The aim of this paper is to close the capacity gap of the 2user 2-hop networks within a constant number of bits/sec/Hz, independent of SNR and the number of relays. Our achievability is based on ergodic interference neutralization [9], [12],

\footnotetext{
${ }^{\dagger} \mathrm{He}$ is also with the Department of Electrical Engineering and Computer Sciences, University of California, Berkeley, CA, USA.
}

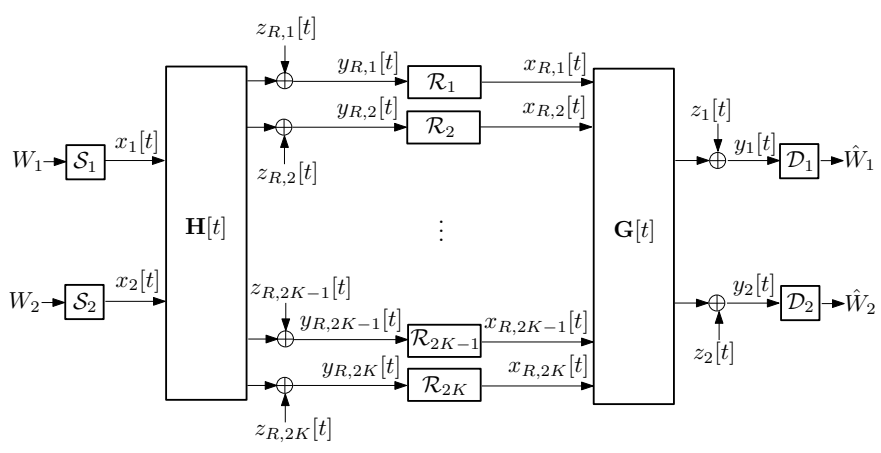

Fig. $1 . \quad 2 \times 2 K \times 2$ networks

which is similar to ergodic interference alignment [5], [13] applied for multi-source single-hop networks. Our previous work [12] considered 2-user 2-hop networks assuming that the number of relays $L$ is equal to 2 and characterized the ergodic sum capacity within $4 \mathrm{bits} / \mathrm{sec} / \mathrm{Hz}$ for independently and identically distributed (i.i.d.) uniform phase fading and approximately $4.7 \mathrm{bits} / \mathrm{sec} / \mathrm{Hz}$ for i.i.d. Rayleigh fading. As the number of relays $L$ increases, the ergodic sum capacity increases approximately as $2 \log L$ and we can narrow the corresponding gap in our analysis. We show that in the limits of large $L$ this gap is given by $4((\log \pi)-1) \simeq 2.6$ for i.i.d. uniform phase fading and $4(4-\log 3 \pi) \simeq 3.1$ for i.i.d. Rayleigh fading.

\section{System Model}

Throughout the paper, we will use $\mathbf{A}$, a, and $\mathcal{A}$ to denote a matrix, vector, and set, respectively. Let $\mathbf{A}^{T}$ (or $\mathbf{a}^{T}$ ) and $\mathbf{A}^{\dagger}$ (or $\mathbf{a}^{\dagger}$ ) denote the transpose and conjugate transpose of $\mathbf{A}$ (or a), respectively. Denote $\mathbf{I}$ and $\mathcal{N}_{\mathbb{C}}\left(\mu, \sigma^{2}\right)$ by the identity matrix and the circularly symmetric complex Gaussian distribution with mean $\mu$ and variance $\sigma^{2}$, respectively.

\section{A. Fading $2 \times 2 K \times 2$ Networks}

We study a 2-user 2 -hop network with $2 K$ relays depicted in Fig. 1 where each source wishes to transmit an independent message to its destination. The input-output relation of the first hop at time $t$ is given by

$$
\mathbf{y}_{R}[t]=\mathbf{H}[t] \mathbf{x}[t]+\mathbf{z}_{R}[t]
$$

where $\mathbf{y}_{R}[t]=\left[y_{R, 1}[t], \cdots, y_{R, 2 K}[t]\right]^{T}$ is the received signal vector of the relays at time $t, \mathbf{H}[t]$ is the $2 K \times 2$ dimensional 
complex channel matrix of the first hop at time $t, \mathbf{x}[t]=$ $\left[x_{1}[t], x_{2}[t]\right]^{T}$ is the transmit signal vector of the sources at time $t$, and $\mathbf{z}_{R}[t]=\left[z_{R, 1}[t], \cdots, z_{R, 2 K}[t]\right]^{T}$ is the noise vector of the relays at time $t$. Similarly, the input-output relation of the second hop at time $t$ is given by

$$
\mathbf{y}[t]=\mathbf{G}[t] \mathbf{x}_{R}[t]+\mathbf{z}[t],
$$

where $\mathbf{y}[t]=\left[y_{1}[t], y_{2}[t]\right]^{T}$ is the received signal vector of the destinations at time $t, \mathbf{G}[t]$ is the $2 \times 2 K$ dimensional complex channel matrix of the second hop at time $t, \mathbf{x}_{R}[t]=\left[x_{R, 1}[t], \cdots, x_{R, 2 K}[t]\right]^{T}$ is the transmit signal vector of the relays at time $t$, and $\mathbf{z}[t]=\left[z_{1}[t], z_{2}[t]\right]^{T}$ is the noise vector of the destinations at time $t$. Let $\mathbf{H}_{k}[t]=$ $\left[\left[h_{2 k-1,1}[t], h_{2 k-1,2}[t]\right]^{T},\left[h_{2 k, 1}[t], h_{2 k, 2}[t]\right]^{T}\right]^{T}$ denote the $2 \times$ 2 dimensional channel matrix at time $t$ from the sources to relays $2 k-1$ and $2 k$, where $h_{i, j}[t]$ is the $(i, j)$ th element of $\mathbf{H}[t]$. Then $\mathbf{H}[t]=\left[\mathbf{H}_{1}[t]^{T}, \cdots, \mathbf{H}_{K}[t]^{T}\right]^{T}$. Similarly, denote $\mathbf{G}_{k}[t]=\left[\left[g_{1,2 k-1}[t], g_{1,2 k}[t]\right]^{T},\left[g_{2,2 k-1}[t], g_{2,2 k}[t]\right]^{T}\right]^{T}$ and $\mathbf{G}[t]=\left[\mathbf{G}_{1}[t], \cdots, \mathbf{G}_{K}[t]\right]$, where $g_{j, i}[t]$ denote the $(j, i)$ th element of $\mathbf{G}[t]$. Each source and relay should satisfy the average power constraint $P$ and the elements of $\mathbf{z}_{R}[t]$ and $\mathbf{z}[t]$ are i.i.d. drawn from $\mathcal{N}_{\mathbb{C}}(0,1)$.

We assume that channel coefficients are i.i.d. drawn from a continuous distribution $f(x), x \in \mathbb{C}$, and vary independently and identically over time. Without loss of generality, we assume that $\mathrm{E}\left[\left|h_{i, j}[t]\right|^{2}\right]=1$ and $\mathrm{E}\left[\left|g_{j, i}[t]\right|^{2}\right]=1$ for all $i \in\{1, \cdots, 2 K\}$ and $j \in\{1,2\}$. We further assume that the sources do not know any channel state information (CSI) and the relays and the destinations know global CSI. That is, at time $t$, each relay and destination knows $\mathbf{H}[t]$ and $\mathbf{G}[t]$.

\section{B. Setup}

Based on the network model, we consider a set of length- $n$ block codes. Let $W_{i}$ be the message of source $i$ uniformly distributed over $\left\{1, \cdots, 2^{n R_{i}}\right\}$, where $R_{i}$ is the rate of source $i$. A rate pair $\left(R_{1}, R_{2}\right)$ is said to be achievable if there exists a sequence of $\left(2^{n R_{1}}, 2^{n R_{2}}, n\right)$ codes such that the probabilities of error for $W_{1}$ and $W_{2}$ converge to zero as $n$ increases. The sum capacity $C_{\text {sum }}$ is defined as the maximum achievable sum rate. In the rest of the paper, we will characterize an approximate capacity on $C_{\text {sum }}$ for a broad class of channel distributions including Rayleigh fading.

\section{Main Results}

In this section, we first introduce our main results and explain the proposed ergodic interference neutralization in the next section.

\section{A. Achievable Sum Rate}

Theorem 1: For the fading $2 \times 2 K \times 2$ network,

$$
R_{i}=\mathrm{E}\left[\log \left(1+\frac{P \gamma^{2}\left(\sum_{k=1}^{K}\left|\operatorname{det}\left(\mathbf{H}_{k}\right)\right|\right)^{2}}{1+\gamma^{2} \sum_{k=1}^{K} a_{k i}}\right)\right]
$$

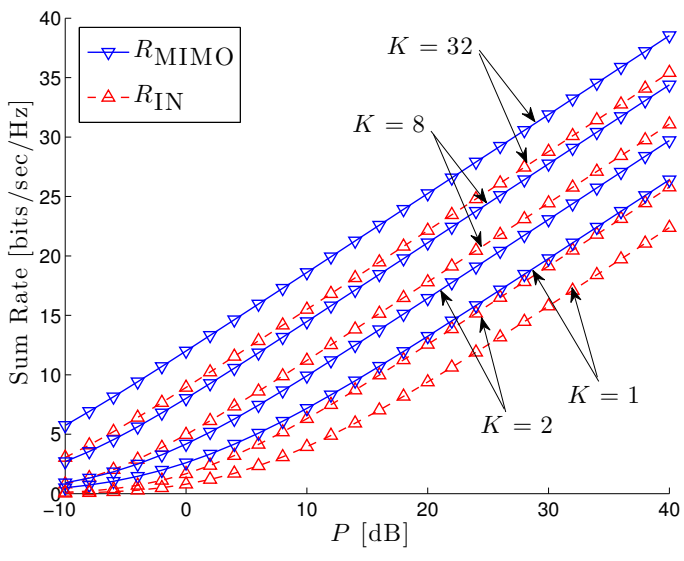

Fig. 2. Achievable sum rates for i.i.d. Rayleigh fading, where $K=$ $1,2,8,32$.

is achievable for $i \in\{1,2\}$, where the expectation is over the channel coefficients. ${ }^{1}$ Here $\gamma=\sqrt{\frac{P}{1+2 P}}$ and $a_{k i}=$ $\left|h_{2(k-1)+j, j}\right|^{2}+\left|h_{2(k-1)+i, j}\right|^{2}, j=3-i$.

Remark 1: Theorem 1 generalizes our previous result in [12], which corresponds to the case where $K=1$. Therefore the proposed ergodic interference neutralization characterizes $C_{\text {sum }}$ within $4 \mathrm{bits} / \mathrm{sec} / \mathrm{Hz}$ for i.i.d. uniform phase fading and approximately $4.7 \mathrm{bits} / \mathrm{sec} / \mathrm{Hz}$ for i.i.d. Rayleigh fading when $K=1$. We refer to [12] and also the full paper [14] in preparation for the proof.

For convenience, let $R_{\mathrm{IN}}$ denote the achievable sum rate of Theorem 1. Since channel coefficients are i.i.d., $C_{\text {sum }}$ is upper bounded by the ergodic capacity of the multipleinput multiple-output (MIMO) channel from the sources to the relays, that is

$$
R_{\mathrm{MIMO}}:=\mathrm{E}\left[\log \operatorname{det}\left(\mathbf{I}+P \mathbf{H H}^{\dagger}\right)\right] .
$$

Example 1 (Sum rate: Rayleigh fading): Figure 2 plots $R_{\mathrm{MIMO}}$ and $R_{\mathrm{IN}}$ for i.i.d. Rayleigh fading, i.e., $f(x)$ follows $\mathcal{N}_{\mathbb{C}}(0,1)$. As shown in the figure, $R_{\mathrm{MIMO}}-R_{\mathrm{IN}}$ decreases as $K$ increases. Apparently the proposed ergodic interference neutralization can narrow the gap from $C_{\text {sum }}$ as the number of relays increases.

\section{B. Approximate Capacity}

In this subsection, we consider a class of channel distributions such that $f(x)$ is only a function of its magnitude $|x|$. That is, for a given channel amplitude, its phase is uniformly distributed over $[0,2 \pi)$ independent of the channel amplitude. We derive an upper bound on the gap between the ergodic sum capacity and the achievable lower bound in Theorem 1 in the limit of large $K$.

\footnotetext{
${ }^{1}$ Since the channel coefficients are i.i.d. over time, we omit the time index for notational simplicity.
} 


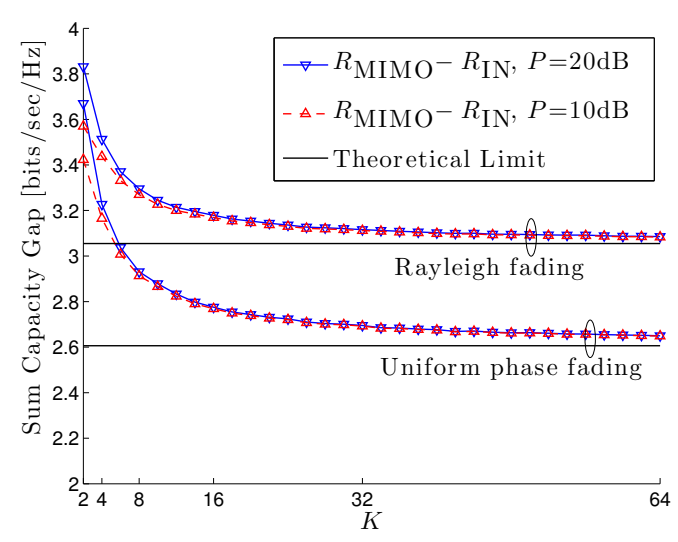

Fig. 3. Gap from the sum capacity for i.i.d. uniform phase fading and i.i.d Rayleigh fading.

In the following theorem, we first characterize the sum capacity gap for i.i.d. uniform phase fading.

Theorem 2: Consider the fading $2 \times 2 K \times 2$ network. If $h_{i, j}[t]=\exp \left(\jmath \theta_{i, j}[t]\right), g_{j, i}[t]=\exp \left(\jmath \varphi_{j, i}[t]\right)$, and $\theta_{i, j}[t]$ and $\varphi_{j, i}[t]$ are uniformly distributed over $[0,2 \pi)$ for all $i \in\{1, \cdots, 2 K\}$ and $j \in\{1,2\}$, then

$$
\lim _{K \rightarrow \infty}\left\{C_{\text {sum }}-R_{\mathrm{IN}}\right\} \leq 4((\log \pi)-1) .
$$

Example 2 (Gap as $K \rightarrow \infty$ : Uniform phase fading): Figure 3 plots the gap $R_{\mathrm{MIMO}}-R_{\mathrm{IN}}$ for i.i.d. uniform phase fading with respect to $K$. As shown in the figure, the gaps decrease as $K$ increases and eventually converge to $4((\log \pi)-1)$ regardless of $P$, which was proved in Theorem 2. Therefore the proposed ergodic interference alignment characterizes $C_{\text {sum }}$ within $4((\log \pi)-1) \mathrm{bits} / \mathrm{sec} / \mathrm{Hz}$ in the limit of large $K$. Compared to $4 \mathrm{bits} / \mathrm{sec} / \mathrm{Hz}$, the sum capacity gap when $K=1$ (Remark 1), the sum capacity gap can be tightened as $K$ increases.

In the following theorem, we characterize the sum capacity gap assuming that $f(x)$ is only a function of $|x|$. The presented gap only depends on the channel amplitude distribution and does not depend on the transmit power constraint $P$.

Theorem 3: Consider the fading $2 \times 2 K \times 2$ network. If $f(x)$ is only a function of $|x|$, then

$$
\lim _{K \rightarrow \infty}\left\{C_{\text {sum }}-R_{\mathrm{IN}}\right\} \leq 4-4 \log \left(\mathrm{E}\left[\left|\operatorname{det}\left(\mathbf{H}_{1}\right)\right|\right]\right) .
$$

Example 3 (Gap as $K \rightarrow \infty$ : Rayleigh fading): Figure 3 plots $R_{\mathrm{MIMO}}-R_{\mathrm{IN}}$ for i.i.d. Rayleigh fading with respect to $K$. That is, $f(x)$ follows $\mathcal{N}_{\mathbb{C}}(0,1)$. It can be shown that $\mathrm{E}\left[\left|\operatorname{det}\left(\mathbf{H}_{1}\right)\right|\right]=3 \pi / 8$ for i.i.d. Rayleigh fading and thus the theoretical limit in Theorem 3 is $4(4-\log 3 \pi) \simeq 3.1$. As shown in the figure, $R_{\mathrm{MIMO}}-R_{\mathrm{IN}}$ quickly converges to the theoretical limit as $K$ increases. Considering that the sum capacity gap is approximately given by $4.7 \mathrm{bits} / \mathrm{sec} / \mathrm{Hz}$ when $K=1$ (Remark 1 ), the sum capacity gap can be tightened as $K$ increases.
Remark 2: The same bounds in Theorems 2 and 3 hold for a general number of relays $L$. We can simply use $2\lfloor L / 2\rfloor$ relays and apply the proposed ergodic interference neutralization, which gives the same bounds in the limit of large $L$, where $\lfloor x\rfloor=\max \{a \in \mathbb{Z} \mid a \leq x\}$.

\section{ERgodic Interference NeUtralization}

In this section, we propose ergodic interference neutralization for fading $2 \times 2 K \times 2$ networks.

\section{A. Main idea}

As pointed out in [9], [12], a simple amplify-and-forward with an appropriate delay $\tau$ is enough to neutralize interference at both destinations if $\mathbf{G}[t+\tau] \mathbf{H}[t]$ approximately becomes a diagonal matrix with non-zero diagonal elements. In this paper, we impose a block-wise interference neutralization condition such that $\mathbf{G}_{k}[t+\tau] \mathbf{H}_{k}[t]$ approximately becomes a diagonal matrix for all $k \in\{1, \cdots, K\}$. Then for each block $k$ we apply the same channel pairing proposed in [14]. Specifically, for $\mathbf{A}=\left[\left[a_{1,1}, a_{1,2}\right]^{T},\left[a_{2,1}, a_{2,2}\right]^{T}\right]^{T} \in \mathbb{C}^{2 \times 2}$, define

$$
F_{2}(\mathbf{A})=\left[\begin{array}{ll}
a_{2,2} & a_{1,2} \\
a_{2,1} & a_{1,1}
\end{array}\right] \text {. }
$$

The relays amplify and forward $\mathbf{y}_{R}[t]$ with appropriate delay $\tau$ if $\mathbf{G}_{k}[t+\tau]$ is approximately given by $F_{2}\left(\mathbf{H}_{k}[t]\right)$ for all $k$. For relaying, the first and the second relays in the $k$ th block (relays $2 k-1$ and $2 k$, respectively) amplify and forward with the amplification coefficients $\gamma \frac{\operatorname{det}\left(\mathbf{H}_{k}[t]\right)^{*}}{\left|\operatorname{det}\left(\mathbf{H}_{k}[t]\right)\right|}$ and $-\gamma \frac{\operatorname{det}\left(\mathbf{H}_{k}[t]\right)^{*}}{\left|\operatorname{det}\left(\mathbf{H}_{k}[t]\right)\right|}$, respectively. Hence this scheme guarantees that the effective channel matrix of the $k$ th block is approximately given by

$$
\gamma \frac{\operatorname{det}\left(\mathbf{H}_{k}[t]\right)^{*}}{\left|\operatorname{det}\left(\mathbf{H}_{k}[t]\right)\right|} \mathbf{G}_{k}[t+\tau]\left[\begin{array}{cc}
1 & 0 \\
0 & -1
\end{array}\right] \mathbf{H}_{k}[t]=\gamma\left|\operatorname{det}\left(\mathbf{H}_{k}[t]\right)\right| \mathbf{I}
$$

which provides the overall effective channel matrix from the sources to the destinations as $\left(\sum_{k=1}^{K} \gamma\left|\operatorname{det}\left(\mathbf{H}_{k}[t]\right)\right|\right) \mathbf{I}$. Here, $\gamma=\sqrt{\frac{P}{1+2 P}}$ is the power amplification factor to satisfy the average power constraint $P$ and $\frac{\operatorname{det}\left(\mathbf{H}_{k}[t]\right)^{*}}{\left|\operatorname{det}\left(\mathbf{H}_{k}[t]\right)\right|}$ is needed to compensate the phase of the $k$ th effective channel matrix guaranteeing coherent combining gain over the blocks. As a result, the overall channel gain $\gamma^{2}\left(\sum_{k=1}^{K}\left|\operatorname{det}\left(\mathbf{H}_{k}[t]\right)\right|\right)^{2}$ appears in the numerator of Theorem 1. The term $\gamma^{2} \sum_{k=1}^{K} a_{k i}$ in the denominator of Theorem 1 is the additional noise due to amplify-and-forward relaying. Lastly, since the probability distributions for $\left[\mathbf{H}_{1}[t]^{T}, \cdots, \mathbf{H}_{K}[t]^{T}\right]^{T}$ at the first hop and $\left[F\left(\mathbf{H}_{1}[t]\right), \cdots, F\left(\mathbf{H}_{K}[t]\right)\right]$ at the second hop are the same, we can utilize almost all channel instances, which provides Theorem 1.

Although finding a pair of channel instances having exact prescribed values is impossible, such a pairing can be done approximately by partitioning the channel space of each hop. In the following two subsections, we first explain channel space partition and pairing and then explain the detailed proposed scheme. 


\section{B. Partitioning and Pairing of Channel Space}

We partition the channel space of each hop, i.e, $\mathbb{C}^{2 K \times 2}$ space for the first hop and $\mathbb{C}^{2 \times 2 K}$ space for the second hop. For $M \in \mathbb{Z}_{+}$and $\Delta>0$, define $\mathcal{Q}_{1}=\left\{\mathbf{A} \in \Delta\left(\mathbb{Z}^{2 K \times 2}+\right.\right.$ $\left.\jmath \mathbb{Z}^{2 K \times 2}\right)|| \operatorname{re}\left(a_{i, j}\right)|\leq \Delta M,| \operatorname{im}\left(a_{i, j}\right) \mid \leq \Delta M$ for all $i \in$ $\{1, \cdots, 2 K\}$ and $j \in\{1,2\}\}$, where $a_{i, j}$ denotes the $(i, j)$ th element of $\mathbf{A}$ and $\mathrm{re}(\cdot)$ and $\operatorname{im}(\cdot)$ denote the real and imaginary parts of a complex number. Here, $M$ and $\Delta$ are related to the number of quantization points and the quantization interval. For a quantized channel matrix $\mathbf{Q} \in \mathcal{Q}_{1}$, define $\mathcal{A}_{1}(\mathbf{Q})=$ $\left\{\mathbf{A} \in \mathbb{C}^{2 K \times 2} \mid-\frac{\Delta}{2} \leq \operatorname{re}\left(a_{i, j}\right)-\operatorname{re}\left(q_{i, j}\right)<\frac{\Delta}{2}\right.$ and $-\frac{\Delta}{2} \leq$ $\operatorname{im}\left(a_{i, j}\right)-\operatorname{im}\left(q_{i, j}\right)<\frac{\Delta}{2}$ for all $i \in\{1, \cdots, 2 K\}$ and $j \in$ $\{1,2\}\}$, where $a_{i, j}$ and $q_{i, j}$ denote the $(i, j)$ th element of $\mathbf{A}$ and $\mathbf{Q}$, respectively. We will use $\mathcal{A}_{1}(\mathbf{Q})$ for partitioning the first-hop channel space. Similarly, we can define $\mathcal{Q}_{2}$ and $\mathcal{A}_{2}(\mathbf{Q})$ for the second hop.

Now consider the channel space pairing. For $\mathbf{A} \in \mathbb{C}^{2 K \times 2}$, define $F(\mathbf{A})=\left[F_{2}\left[\mathbf{A}_{1}\right], F_{2}\left[\mathbf{A}_{2}\right], \cdots, F_{2}\left[\mathbf{A}_{K}\right]\right]$, where $\mathbf{A}=$ $\left[\mathbf{A}_{1}^{T}, \mathbf{A}_{2}^{T}, \cdots, \mathbf{A}_{K}^{T}\right]^{T}$ and the definition of $F_{2}(\cdot)$ is given by (4). Note that $F(\mathbf{Q}) \in \mathcal{Q}_{2}$ for any $\mathbf{Q} \in \mathcal{Q}_{1}$. The relays will choose a certain time $t_{2}$ and amplify and forward $\mathbf{y}_{R}\left[t_{1}\right]$ such that $\mathbf{H}\left[t_{1}\right] \in \mathcal{A}_{1}(\mathbf{Q})$ and $\mathbf{G}\left[t_{2}\right] \in \mathcal{A}_{2}(F(\mathbf{Q}))$. Hence the channel subspace $\mathcal{A}_{1}(\mathbf{Q})$ of the first hop will be paired with the channel subspace $\mathcal{A}_{2}(F(\mathbf{Q}))$ of the second hop.

Based on the channel space partition and pairing rule described in this subsection, we propose ergodic interference neutralization in the next subsection.

\section{Block-wise Interference Neutralization}

We first divide a length- $n$ block into $B$ sub-blocks having length $n_{B}=\frac{n}{B}$ each. We assume block transmission. At the first sub-block, the sources transmit their first messages to the relays (the relays do not transmit). At the bth sub-block, $b \in$ $\{2, \cdots, B-1\}$, the sources transmit their $b$ th messages to the relays and the relays amplify and forward the received signals of the $(b-1)$ th sub-block to the destinations. At the last subblock, the relays amplify and forward the received signals of the $(B-1)$ th sub-block to the destinations (the sources do not transmit). Hence, the number of effective sub-blocks is equal to $B-1$. Since we can set both $n_{B}$ and $B$ as large as possible as $n$ increases, the fractional rate loss $\frac{1}{B}$ becomes negligible as $n$ increases. For simplicity, we describe the proposed scheme based on the first message transmission and omit the sub-block index.

For $\mathbf{Q} \in \mathcal{Q}_{1}$, denote $\mathcal{T}_{1}(\mathbf{Q})=\left\{t \mid \mathbf{H}[t] \in \mathcal{A}_{1}(\mathbf{Q}), t \in\right.$ $\left.\left\{1, \cdots, n_{B}\right\}\right\}$, which is the set of time indices of the first hop whose channel instances belong to $\mathcal{A}_{1}(\mathbf{Q})$. Similarly, for $\mathbf{Q} \in$ $\mathcal{Q}_{2}$, denote $\mathcal{T}_{2}(\mathbf{Q})=\left\{t \mid \mathbf{G}[t] \in \mathcal{A}_{2}(\mathbf{Q}), t \in\left\{1, \cdots, n_{B}\right\}\right\}$, which is the set of time indices of the second hop whose channel instances belong to $\mathcal{A}_{2}(\mathbf{Q})$. The encoding, relaying, and decoding are as follows.

- (Encoding) The sources transmit their messages using Gaussian codebook with power $P$. Specifically, the transmit signal vector of the sources is given by $\mathbf{x}[t]$ with $\mathrm{E}\left[\mathbf{x}[t] \mathbf{x}[t]^{\dagger}\right]=P \mathbf{I}$ for $t \in\left\{1, \cdots, n_{B}\right\}$.
- (Relaying) For all $\mathbf{Q} \in \mathcal{Q}_{1}$, the relays amplify and forward their received signals that were received during $\mathcal{T}_{1}(\mathbf{Q})$ using the time indices in $\mathcal{T}_{2}(F(\mathbf{Q}))$. Specifically, the transmit signal vector of the relays is given by $\mathbf{x}_{R}\left[t_{2}\right]=\boldsymbol{\Gamma}(\mathbf{Q}) \mathbf{y}_{R}\left[t_{1}\right]$, where $t_{1} \in \mathcal{T}_{1}(\mathbf{Q})$, and $t_{2} \in$ $\mathcal{T}_{2}(F(\mathbf{Q}))$. Here

$$
\boldsymbol{\Gamma}(\mathbf{Q})=\left[\begin{array}{cccc}
\gamma \boldsymbol{\Lambda}_{1} & \mathbf{0} & \cdots & \mathbf{0} \\
\mathbf{0} & \gamma \boldsymbol{\Lambda}_{2} & & \vdots \\
\vdots & & \ddots & \\
\mathbf{0} & \cdots & & \gamma \boldsymbol{\Lambda}_{K}
\end{array}\right],
$$

$\gamma=\sqrt{\frac{P}{1+2 P}}$, and $\boldsymbol{\Lambda}_{k}=\frac{\operatorname{det}\left(\mathbf{Q}_{k}\right)^{*}}{\left|\operatorname{det}\left(\mathbf{Q}_{k}\right)\right|}\left[[1,0]^{T}[0,-1]^{T}\right]^{T}$, where $\mathbf{Q}=\left[\mathbf{Q}_{1}^{T}, \cdots, \mathbf{Q}_{K}^{T}\right]^{T}$ and $\mathbf{0}$ denotes the $(2 \times 2)$ all-zero matrix.

- (Decoding) The destinations decode their messages based on their received signals for $t \in\left\{1, \cdots, n_{B}\right\}$.

Then we can set $\Delta \rightarrow 0$ and $M \rightarrow \infty$, which are functions of $n_{B}$, such that the rate in Theorem 1 is achievable. We refer to the full paper [14] in preparation for the detailed proof of Theorem 1 and also refer to the paper [12], which is the case where $K=1$.

\section{APPROXIMATE CAPACITY CHARACTERIZATION}

In this section, we prove Theorems 2 and 3. For $N$-user 2-hop networks with $L$ relays, it was shown in [7] that interference can be completely neutralized if $L \geq N(N-$ 1) +1 , which indicates that for $2 \times L \times 2$ networks with $L \geq 3$ interference neutralization can be achieved without channel pairing. However, maximizing the achievable sum rate exploiting interference neutralization without channel pairing presented in [15] is non-convex and, as a result, it is unclear how to determine the sum rate gap from the cut-set upper bound. By contrast, we now show that our achievable rate expression from Theorem 1 permits to derive a finite-gap result.

We first introduce the following lemma, which will be used to prove Theorems 2 and 3.

Lemma 1: Consider a sequence of i.i.d. nonnegative random variables $\left\{X_{m}, m \in \mathbb{N}\right\}$. Let $S_{m}=\sum_{i=1}^{m} X_{i}$. If $\mathrm{E}\left[X_{1}^{2}\right]<\infty$, then for any $\epsilon \in\left(0, \mathrm{E}\left[X_{1}\right]\right)$ and any $c>0$,

$$
\begin{aligned}
& \mathrm{E}\left[\log \left(1+c S_{m}^{2}\right)\right] \\
\geq & \log \left(1+c m^{2}\left(\mathrm{E}\left[X_{1}\right]\right)^{2}\right)-\delta_{m}\left(c, \mathrm{E}\left[X_{1}\right], \mathrm{E}\left[X_{1}^{2}\right]\right),
\end{aligned}
$$

where

$$
\begin{aligned}
\delta_{m}\left(c, \mathrm{E}\left[X_{1}\right], \mathrm{E}\left[X_{1}^{2}\right]\right) & =\frac{\mathrm{E}\left[X_{1}^{2}\right]}{m \epsilon^{2}} \log \left(1+c m^{2}\left(\mathrm{E}\left[X_{1}\right]-\epsilon\right)^{2}\right) \\
& -\log \left(1-\frac{c m^{2} \epsilon\left(2 \mathrm{E}\left[X_{1}\right]-\epsilon\right)}{1+c m^{2}\left(\mathrm{E}\left[X_{1}\right]\right)^{2}}\right)>0 .
\end{aligned}
$$

By setting $\delta_{m}$ arbitrarily small, Lemma 1 approximately provides that $\mathrm{E}\left[\log \left(1+c S_{m}^{2}\right)\right] \geq \log \left(1+c m^{2}\left(\mathrm{E}\left[X_{1}\right]\right)^{2}\right)$ as $m$ increases. This bound is tight in the limit of large $m$ since $\mathrm{E}\left[\log \left(1+c{S_{m}}^{2}\right)\right] \leq \log \left(1+c \mathrm{E}\left[{S_{m}}^{2}\right]\right)$ from Jensen's 
inequality and $\log \left(1+c \mathrm{E}\left[S_{m}^{2}\right]\right)$ is approximately given by $\log \left(1+c m^{2} \mathrm{E}\left[X_{1}\right]^{2}\right)$ as $m$ increases.

\section{A. Proof of Theorem 2}

From (3), we have

$$
C_{\text {sum }} \leq R_{\mathrm{MIMO}} \leq 2 \log (1+2 P K)
$$

where the second inequality follows from Jensen's inequality and the fact that $\log \operatorname{det}(\cdot)$ is a concave function [16]. Also,

$$
\begin{aligned}
& R_{\mathrm{IN}} \\
& \stackrel{(a)}{=} 2 \mathrm{E}_{\left\{\theta_{1}, \cdots, \theta_{K}\right\}}\left[\log \left(1+\frac{P^{2}\left(\sum_{k=1}^{K} \sqrt{2-2 \cos \theta_{k}}\right)^{2}}{1+P(2 K+2)}\right)\right] \\
& \stackrel{(b)}{\geq} 2 \log \left(1+\frac{\frac{16}{\pi^{2}} P^{2} K^{2}}{1+P(2 K+2)}\right) \\
& \quad-2 \delta_{K}\left(\frac{P^{2}}{1+P(2 K+2)}, \frac{4}{\pi}, 2\right)
\end{aligned}
$$

where $\theta_{k}=\theta_{2 k-1,1}+\theta_{2 k, 2}-\theta_{2 k-1,2}-\theta_{2 k, 1}$. Here, $(a)$ follows since $\left|\operatorname{det}\left(\mathbf{H}_{k}\right)\right|=\sqrt{2-2 \cos \theta_{k}}$ and $(b)$ follows since $\theta_{k} \bmod [2 \pi]$ is uniformly distributed over $[0,2 \pi)$ and from Lemma 1 with the facts that $E\left[\sqrt{2-2 \cos \phi_{1}}\right]=\frac{4}{\pi}$, and $E\left[2-2 \cos \phi_{1}\right]=2$. Hence

$$
\begin{aligned}
C_{\text {sum }}-R_{\mathrm{IN}} \leq & 2 \log \left(\frac{1+P(4 K+2)+P^{2} 2 K(2 K+2)}{1+P(2 K+2)+\frac{16}{\pi^{2}} P^{2} K^{2}}\right) \\
& +2 \delta_{K}\left(\frac{P^{2}}{1+P(2 K+2)}, \frac{4}{\pi}, 2\right)
\end{aligned}
$$

and $\lim _{K \rightarrow \infty}\left\{C_{\text {sum }}-R_{\mathrm{IN}}\right\} \leq 4((\log \pi)-1)+\epsilon_{1}$, where $\epsilon_{1}=\lim _{K \rightarrow \infty} 2 \delta_{K}=-2 \log \left(1-\epsilon\left(\frac{\pi}{2}-\frac{\pi^{2}}{16} \epsilon\right)\right)>0$, which can be arbitrarily small as $\epsilon$ decreases. Therefore, Theorem 2 holds.

\section{B. Proof of Theorem 3}

From (5), $C_{\text {sum }} \leq 2 \log (1+2 P K)$. Also, $R_{\mathrm{IN}}$ is given as

$$
\begin{aligned}
R_{\mathrm{IN}} & \stackrel{(a)}{=} 2 \mathrm{E}\left[\log \left(1+\frac{P^{2}\left(\sum_{k=1}^{K}\left|\operatorname{det}\left(\mathbf{H}_{k}\right)\right|\right)^{2}}{1+P\left(\sum_{k=1}^{2 K}\left|h_{k, 1}\right|^{2}+2\right)}\right)\right] \\
\geq & 2 \mathrm{E}\left[\log \left(1+P^{2}\left(\sum_{k=1}^{K} \mid \operatorname{det}\left(\mathbf{H}_{k}\right)\right)^{2}\right)\right] \\
& -2 \mathrm{E}\left[\log \left(1+P\left(\sum_{k=1}^{2 K}\left|h_{k, 1}\right|^{2}+2\right)\right)\right] \\
& (b) 2 \log \left(\frac{\left.1+P^{2} K^{2}\left(\mathrm{E}\left[\left|\operatorname{det}\left(\mathbf{H}_{1}\right)\right|\right]\right)^{2}\right)}{1+2 P(K+1)}\right) \\
& -2 \delta_{K}\left(P^{2}, \mathrm{E}\left[\left|\operatorname{det}\left(\mathbf{H}_{1}\right)\right|\right], \mathrm{E}\left[\left|\operatorname{det}\left(\mathbf{H}_{1}\right)\right|^{2}\right]\right)
\end{aligned}
$$

where $(a)$ follows since $\gamma_{k}=\sqrt{\frac{P}{1+2 P}}$ and $(b)$ follows from Lemma 1 and Jensen's inequality. Hence

$$
\begin{aligned}
C_{\text {sum }}-R_{\mathrm{IN}} \leq & 2 \log \left(\frac{1+2 P(2 K+1)+4 P^{2} K(K+1)}{1+P^{2} K^{2}\left(\mathrm{E}\left[\left|\operatorname{det}\left(\mathbf{H}_{1}\right)\right|\right]\right)^{2}}\right) \\
& +2 \delta_{K}\left(P^{2}, \mathrm{E}\left[\left|\operatorname{det}\left(\mathbf{H}_{1}\right)\right|\right], \mathrm{E}\left[\left|\operatorname{det}\left(\mathbf{H}_{1}\right)\right|^{2}\right]\right)
\end{aligned}
$$

and

$$
\lim _{K \rightarrow \infty}\left\{C_{\text {sum }}-R_{\mathrm{IN}}\right\} \leq 4-4 \log \left(\mathrm{E}\left[\left|\operatorname{det}\left(\mathbf{H}_{1}\right)\right|\right]\right)+\epsilon_{2},
$$

where $\epsilon_{2}=\lim _{K \rightarrow \infty} 2 \delta_{K}=-2 \log \left(1-\frac{\epsilon\left(2 \mathrm{E}\left[\left|\operatorname{det}\left(\mathbf{H}_{1}\right)\right|\right]-\epsilon\right)}{\left(\mathrm{E}\left[\left|\operatorname{det}\left(\mathbf{H}_{1}\right)\right|\right]\right)^{2}}\right)$ $>0$, which can be arbitrarily small as $\epsilon$ decreases. Therefore, Theorem 3 holds.

\section{ACKNOWLEDGEMENT}

This work has been supported in part by the European ERC Starting Grant 259530-ComCom.

\section{REFERENCES}

[1] R. H. Etkin, D. Tse, and H. Wang, "Gaussian interference channel capacity to within one bit," IEEE Trans. Inf. Theory, vol. 54, pp. 55345562, Dec. 2008

[2] G. Bresler, A. Parekh, and D. Tse, "The approximate capacity of the many-to-one and one-to-many Gaussian interference channels," IEEE Trans. Inf. Theory, vol. 56, pp. 4566-4592, Sep. 2010.

[3] W. Nam, S.-Y. Chung, and Y. H. Lee, "Capacity of the Gaussian twoway relay channel to within $\frac{1}{2}$ bit," IEEE Trans. Inf. Theory, vol. 56, pp. 5488-5494, Nov. 2010.

[4] V. R. Cadambe and S. A. Jafar, "Interference alignment and degrees of freedom of the $K$-user interference channel," IEEE Trans. Inf. Theory, vol. 54, pp. 3425-3441, Aug. 2008.

[5] B. Nazer, M. Gastpar, S. A. Jafar, and S. Vishwanath, "Ergodic interference alignment," in arXiv:cs.IT/0901.4379, Jan. 2009.

[6] S. Mohajer, S. N. Diggavi, C. Fragouli, and D. Tse, "Approximate capacity of a class of Gaussian interference-relay networks," IEEE Trans. Inf. Theory, vol. 57, pp. 2837-2864, May 2011.

[7] B. Rankov and A. Wittneben, "Spectral efficient protocols for halfduplex fading relay channels," IEEE J. Select. Areas Commun., vol. 25 , pp. 379-389, Fub. 2007.

[8] T. Gou, S. A. Jafar, S.-W. Jeon, and S.-Y. Chung, "Aligned interference neutralization and the degrees of freedom of the $2 \times 2 \times 2$ interference channel," in arXiv:cs.IT/1012.2350, Dec. 2010.

[9] S.-W. Jeon, S.-Y. Chung, and S. A. Jafar, "Degrees of freedom region of a class of multisource Gaussian relay networks," IEEE Trans. Inf. Theory, vol. 57, pp. 3032-3044, May 2011.

[10] C. Wang, T. Gou, and S. A. Jafar, "Multiple unicast capacity of 2-source 2-sink networks," in arXiv:cs.IT/1 104.0954, Apr. 2011.

[11] I. Shomorony and A. S. Avestimehr, "Two-unicast wireless networks: Characterizing the degrees-of-freedom," in arXiv:cs.IT/1102.2498, Feb. 2011.

[12] S.-W. Jeon, C.-Y. Wang, and M. Gastpar, "Approximate ergodic capacity of a class of fading $2 \times 2 \times 2$ networks," in Proc. Information Theory and Applications Workshop, San Diego, CA, Feb. 2012.

[13] S. A. Jafar, "The ergodic capacity of phase-fading interference networks," IEEE Trans. Inf. Theory, vol. 57, pp. 7685-7694, Dec. 2011.

[14] S.-W. Jeon, C.-Y. Wang, and M. Gastpar, "Approximate ergodic capacity of a class of fading 2-user 2-hop networks," in preparation.

[15] C. Esli, S. Berger, and A. Wittneben, "Optimizing zero-forcing based gain allocation for wireless multiuser networks," in Proc. IEEE ICC, Beijing, China, Jun. 2007.

[16] S. Boyd and L. Vandenberghe, Convex Optimization. New York: Cambridge Univ. Press, 2004. 